\title{
EVALUATION OF MIXED FREQUENCY APPROACHES FOR TRACKING NEAR-TERM ECONOMIC DEVELOPMENTS IN NORTH MACEDONIA
}

Gani Ramadani, Magdalena Petrovska, Vesna Bucevska

\section{Abstract}

Aggregate demand forecasting, also known as nowcasting when it applies to current quarter assessment, is of notable interest to policy makers. This paper concentrates on the empirical methods dealing with mixedfrequency data. In particular, it focuses on the MIDAS approach and its later extension, the Bayesian MF$V A R$. The two strategies are evaluated in terms of their accuracy to nowcast Macedonian GDP growth, using same monthly frequency data set. The results of this study indicate that the MIDAS regressions demonstrate comparable forecasting performance to that of MF-VAR model. Moreover, it is interesting to note that the two approaches are reciprocal, since in general, their combined forecast demonstrates clear superiority in predicting business cycle turning points. Additionally, the MF-VAR model showed higher precision in times of increased uncertainty.

Keywords: MF-VAR, Bayesian estimation, MIDAS, forecast pooling, forecast evaluation

JEL Classification: E37, C53

\section{Introduction}

From their sampling frequency perspective, economic data vary considerably. In decision-making process, we are faced with both, the problem of data frequency and the publication delay. In this regard, the State Statistical Office of the Republic of North Macedonia, following the international practices, releases quarterly figure for Gross Domestic Product (GDP) with a delay of slightly more than two months. Having in mind that these data are a crucial asset for establishing and implementing policies, their rough estimation is therefore necessary. Estimation can be based on monthly readings, such as the industrial production volume index, external trade, value indices of turnover in the retail trade, etc. This whole process is known as nowcasting. The fundamental postulate of nowcasting lies in exploiting the information released early and eventually at higher frequency than the variable of interest so that one may get an "early estimate" before the
Gani Ramadani (corresponding author)

Senior Advisor

Monetary Policy and Research Department,

National Bank of the Republic of North Macedonia

E-mail: ramadanig@nbrm.mk

Address: Blvd Kuzman Josifovski Pitu 1,

1000 Skopje, Republic of North Macedonia

ORCID: https://orcid.org/0000-0001-5861-6981

\section{Magdalena Petrovska}

Senior Advisor

Monetary Policy and Research Department,

National Bank of the Republic of North Macedonia

E-mail: petrovskam@nbrm.mk

Vesna Bucevska, PhD

Full professor of Econometrics and Financial

Econometrics

Faculty of Economics-Skopje,

Ss. Cyril and Methodius University

E-mail: vesna.bucevska@eccf.ukim.edu.mk 
official data becomes accessible (Bańbura et al., 2013).

This gives rise to concern of how to quantify empirically the relationships between variables sampled at various frequencies. The most naive approach is to stick with the lowest frequency in the data, but in this case a loss of possibly valuable high-frequency information is unavoidable. However, the recent literature displays a growing interest in the value added that direct modelling of mixed-frequency could provide.

This paper gives an overview of some of the key approaches employed in the field literature to cope with mixed-frequency data: mixed-frequency Vector Autoregression (MF-VAR) in a Bayesian framework, launched by Schorfheide and Song (2015), as well as the less computationally intensive counterpart, i.e. unrestricted mixed-data sampling (U-MIDAS) approach introduced by Foroni, Marcellino, and Schumacher (2011). To this end, our study is exploratory and interpretative in nature. Analogously, the primary research challenge was to verify which of the considered approaches generalises better and is more capable of producing reliable GDP nowcasts. Therefore, two empirically testable statements were defined: by employing MF-VAR approach, accurate and efficient nowcasts of North Macedonia's GDP can be acquired; by employing U-MIDAS approach, accurate and efficient nowcasts of North Macedonia's GDP can be acquired.

To this end, after discussing in a nutshell the two employed modelling methodologies, we proceed comparing them in an exhaustive empirical exercise. Specifically, we revolve around comparison of the resulting models in terms of the proposed predictions, using same high frequency (HF) data set. To this end, the variable of interest is the Macedonian quarterly GDP growth rate.

A theoretical comparison of these two classes of models points out that, U-MIDAS is more parsimonious than MF-VAR, and as a direct forecasting tool displays greater robustness to misspecification (Kuzin, Marcellino, and Schumacher 2011). This study aims at documenting this status. Moreover, we assess whether the forecast accuracy improves when combining these two models.

\section{Literature review}

Ghysels, Santa-Clara and Valkanov (2004) pioneered one of the most competitive univariate tools suited to handle the mixed-frequency data, i.e. the so called mixed-data sampling method (MIDAS). Mixeddata sampling (MIDAS) models operate with time series at various frequencies. In this structure distributed lag polynomials are employed to ensure parsimony in the specifications.

Initially, MIDAS models have been applied in the financial domain (see for instance Ghysels, Santa Clara, and Valkanov (2006). Recently, numerous applications involve the MIDAS approach as a forecasting platform for quarterly GDP, like for example Clements and Galvão (2008) and Clements and Galvão (2009). Later additions are Foroni, Marcellino, and Schumacher (2011); Kuzin, Marcellino and Schumacher (2011); Drechsel and Scheufele (2012a); Andreou, Ghysels, and Kourtellos (2013); Ferrara, Marsilli, and Ortega (2014); Duarte (2014); and Aastveit, Foroni and Ravazzolo (2016), amongst others. Furthermore, Foroni, Marcellino and Stevanovic (2018) show analytically, in Monte Carlo simulations, the relevance of considering the moving average (MA) component in MIDAS and U-MIDAS models thus closing the gap in the respective literature. Andreou et al. (2019), on the other hand show how the group factor context applies to mixed-frequency data panels.

This study also falls into a relatively new and thus increasing body of literature on mixed frequency VAR models that accommodate a state space approach. The main idea assumes reformulation of each lower frequency series into a partially latent high frequency series. The Kalman filter or, the Gibbs sampler in a Bayesian framework, then allow a partially latent VAR process to be estimated. See Mittnik and Zadrozny (2005); Kuzin, Marcellino and Schumacher (2011); Bai, Ghysels and Wright (2013); and Foroni and Marcellino (2014) as a leading research on state space type MFVAR models adopting a non-Bayesian version of the Kalman filter. On the other hand, Mariano and Murasawa (2010) have a pioneering contribution for a state space type MF-VAR using the expectation-maximization (EM) algorithm, and Chiu et al. (2011) and Schorfheide and Song (2015) for MF-VARs cast in state space form using the Gibbs sampler (for greater coverage and extension of these literature please refer to Mikosch and Neuwirth 2015).

\section{Data}

With respect to the potential high-frequency indicators to draw from, we consider a broad framework of time series routinely employed in the process of GDP nowcasting, starting from economic sentiment indicators to hard data ${ }^{1}$. The selection process was intended to bring about the "best" subset of predictors. To this end, all of the variables were subject to prefiltering based on vigorous one-by-one testing within the bridge 2 equations set-up as a naïve approach to handle the mixed-frequency data ${ }^{3}$. 
The shortlisted variables chosen from a broader set of similar alternatives are actually those who pass the in-sample selection based on their recent forecasting performance. In other words, the informed variable selection procedure that we follow reflects our metrics based on minimum relative forecasting errors.

In addition, all of the considered variables are seasonally adjusted, as well as transformed ensuring their stationarity (e.g. trending variables are expressed as growth rates). The monthly data releases follow similar timing. This allows us to reproduce the same pattern of missing reading at the end of each recursive sample, so to imitate the data availability in real-time. The ragged-edge overview of the dataset is presented in Table A.1 of the Appendix. In addition, depending on the publication timetable, we assume that data are accessible at the earliest at the month-end.

Furthermore, the quarterly variable that we consider, i.e., the real GDP is obtainable in the third month after the end of the referent quarter. For instance, the GDP reading for 2017Q4 becomes acquirable in March 2018.

\section{Methodology \\ 4.1. MF-VAR methodology}

The methodological explanation covering the MF-VAR model is an adaptation from Claudia Foroni's doctoral thesis which provides a compendium of individual mixed-frequency approaches along with a very intuitive understanding of the differences between them.

In the subsequent paragraphs, we describe the main characteristics of the Bayesian MF-VAR approach, following Schorfheide and Song (2015) as a most quintessential study in the field literature.

Namely, these authors cast a MF-VAR in state space form. Furthermore, in order to conduct Bayesian inference for model parameters and unobserved monthly variables, they make use of Markov chain Monte Carlo (MCMC) methods. Following Foroni and Marcellino (2013), the state equation of the model has a $\operatorname{VAR}(\mathrm{p})$ representation, treating quarterly series as monthly series with missing observations, written as it follows:

Let for all $t_{m}$ the latent month-on-month GDP growth $y_{t_{m}}^{*}$ and the corresponding monthly indicator $x_{t_{m}}$ follow a $\operatorname{VAR}(\mathrm{p})$ process

$$
\begin{aligned}
z_{t_{m}}= & F_{1}(\Phi) z_{t_{m-1}}+F_{c}(\Phi)+v_{t_{m}} \\
& v_{t_{m}} \sim \operatorname{iidN}(0, \Omega(\Sigma))
\end{aligned}
$$

In order to specify the measurement equation, the authors have to define the aggregation equation. Following, Kapetanios, Marcellino and Petrova (2018), taking GDP growth as an example, the disaggregation of the quarterly GDP growth, $y_{t_{m}}$, observed every $t_{m}=$ $3 ; 6 ; 9 ; \ldots ; T_{m}$, into the month-on-month GDP growth, $y_{t_{m}}^{*}$, never observed, is based on the following aggregation equation:

$$
y_{t_{m}=} \frac{1}{3}\left(y_{t_{m}}^{*}+y_{t_{m-1}}^{*}+y_{t_{m-2}}^{*}\right)=\Lambda_{m z} z_{t_{m}}
$$

To this end, the quarterly variable is treated as the three-month average of the monthly process.

Following Foroni and Marcellino (2013), since $y_{t_{m}}$ is observed only every third month, there is a need of a selection matrix that equals the identity matrix if $t_{m}$ corresponds to the last month of the quarter and is empty otherwise. Therefore, the measurement equation can be specified as:

$$
\left(\begin{array}{l}
y_{t_{m}} \\
x_{t_{m}}
\end{array}\right)=M_{t_{m}} \Lambda_{z} z_{t_{m}}
$$

where $\mathrm{M}_{t_{m}}$ is the selection matrix.

The problem of dimensionality is surpassed by introducing of a Minnesota prior that shrinks the VAR coefficients toward univariate random walk representations. (adaptation from Kapetanios, Marcellino and Petrova 2018).

\subsection{The U-MIDAS approach}

MIDAS regressions are perceived as a widespread alternate to the multivariate state-space framework elaborated in the previous sub-section. This econometric technique, as a very general type of ARDL model, is based on both a regression structure and a weight function which tracks the high frequency lags of the regressors (Marsilli 2014). The majority of the formulas and back up explanations employed in this section were adapted from Barsoum and Stankiewicz (2013).

The elementary form of the MIDAS ${ }^{4}$ model employed to get an $\mathrm{h}$-step ahead forecast might be expressed following Clements and Galvão (2008):

$$
y_{t}^{Q}=\beta_{0}+\beta_{1} B\left(L^{1 / m} ; \theta\right) x_{t-h}^{M}+\varepsilon_{t}
$$

Where $B\left(L^{1 / m} ; \theta\right)=\sum_{k=0}^{K-1} b(k ; \theta) L^{(k) / m}$ is the sum of weights assigned to $K$ lags of the independent variable (the lag polynomial). $b(k ; \theta)$ is the $\mathrm{k}^{\text {th }}$ weight of the K-lag polynomial, shaped by a certain function of $\theta$ parameters (as for instance an exponential function). $L$ denotes the lag operator so that 
$L^{k / m} x_{t-h}^{M}=x_{t-h-k / m}^{M} . t$ is the time index for $y$, as a lower frequency variable, while $m$ is the time index for the variable with higher frequency, i.e. $x$. $Q$ describes variables observed on a quarterly and $\mathrm{M}$ on a monthly basis.

Having in mind the non-linearity of the lag polynomials, the non-linear least square (NLS) is a typical estimation method for MIDAS models.

However, in some instances the form of the lag polynomial may be overly restrictive in comparison with the underlying data generating process. Thus a model with no restrictions on the weights of the lag polynomial was launched by Foroni, Marcellino and Schumaher (2011) (adaptation from Bersoum and Stankiewicz 2013). These authors advanced a new parametrization scheme for the MIDAS based on a linearization of the distributed lag function called unrestricted MIDAS (U-MIDAS), where all the parameters are estimated using OLS. The U-MIDAS model exploit a linear lag polynomial that can be expressed as (adaptation from Marsilli 2014):

$$
y_{t}^{Q}=\beta_{0}+\sum_{k=0}^{K-1} \beta_{k+1} x_{t-h-k / m}^{M}+\varepsilon_{t}
$$

The dependent variable $y$ is represented by an equation that contains an intercept $\beta_{0}$ and a lag polynomial weighted by parameters $\beta_{k+1}$. To this end, all the parameters $\beta_{k+1}$ of this polynomial need to be estimated as no structure is set on the shape of the weights of the lag polynomial (adaptation from Bersoum and Stankiewicz 2013).

The lags of the explanatory variable are represented by the measure $m$, where $m=3$ specifies the number of observations of the higher-frequency indicator (e.g. monthly variable $x$ ) for each observation of the lower-frequency variable (e.g. quarterly variable $y$ ). That is, if e.g. $y_{\mathrm{t}}$ is the reading of the dependent variable for the first quarter of 2017 (March 2017), then $x_{\mathrm{t}-1}$ represents the observation of the explanatory variable for December 2016 (1 quarter before), $x_{\mathrm{t}-4 / 3}$ for November 2016 (4 months before), whereas $x_{\mathrm{t}-14 / 3}$ for January 2016 (14 months before) and so forth (Bersoum and Stankiewicz 2013).

One of the obvious setbacks of the U-MIDAS lies in the fact that, when the discrepancy in frequencies between the variables in the model is large, its performance plunges significantly due to the rapid increase of the number of parameters. To this end, this method do not fit all kinds of empirical applications (adaptation from Bersoum and Stankiewicz 2013). However, for many macroeconomic analyses the use of the U-MIDAS model may be advantageous.
As we are set to do GDP forecasting, we want to include autoregressive elements in the U-MIDAS model. Simply introducing an AR part into the previous regression we obtain

$$
y_{t}^{Q}=\beta_{0}+\lambda y_{t-1}+\sum_{k=0}^{K-1} \beta_{k+1} x_{t-h-k / m}^{M}+\varepsilon_{t}
$$

Each of the U-MIDAS regressions employed to predict Macedonian real GDP growth, for up to one quarter ahead (i.e., a "nowcast" of the current quarter) use single indicator. With only one indicator in each representation and a restricted number of lags, the coefficients in equation above can be estimated without internalising the degrees of freedom problem (adaptation from Leboeuf and Morel 2014).

Our U-MIDAS specification includes 3 lags of the monthly variables in total, extending over the quarter for which we have the last reading of real GDP growth as well as data along the first quarter to forecast, provided they are obtainable. As the separate monthly readings of the regressors are published over a quarter, the model representation changes slightly (adaptation from Leboeuf and Morel 2014).

In what follows, we provide a real-time demonstration of the U-MIDAS model for the Macedonian GDP as an analogy to the example of the U-MIDAS for euro-area GDP employed by Leboeuf and Morel (2014).

Let $X t^{\left(M^{1)}\right.}, X t^{\left(M^{2)}\right)}$ and $X t^{\left(M^{3)}\right.}$ be monthly variables in the first, second and third month of quarter $t$, for which we are generating a nowcast of $Y t^{(Q)}$ (real GDP growth in quarter $t)$. In other words, $X t^{(M 1)}$ is a quarterly time series containing all first monthly values of the variable $X$ for each quarter over past horizon. $X t^{\left(M^{2}\right)}$ is a quarterly time series encompassing all second monthly values of a variable $X$ for each quarter over past horizon. $X t^{\left(M^{3}\right)}$ is a quarterly time series including all third monthly values of the variable $X$ for each quarter over past horizon (adaptation from Leboeuf and Morel 2014).

For the U-MIDAS approach now we have monthly variables to be transformed into quarterly variables. As in one quarter there are 3 months, each monthly indicator will be transformed into 3 variables with quarterly frequency.

$X t^{\left(M^{1)}\right)}$ - only data for months $1,4,7,10$ are taken

$X t^{\left(M^{2)}\right)}$ - only data for months $2,5,8,11$ are taken

$X t^{\left(M^{3}\right)}$ - only data for months $3,6,9,12$ are taken

Then, just for an illustration, if $X$ has a 1 month publication delay:

- In the first month, the model for $Y$ nowcasting contains a constant, one lag of $Y$ and 3 months of data on variable $X$ 


$$
\begin{gathered}
Y_{t}^{(Q)}=\beta_{1}{ }^{\left(M^{1}\right)}+\varphi_{1} Y_{t-1}{ }^{(Q)}+ \\
\gamma_{2,1} X_{t-1}{ }^{\left(M^{11)}\right.}+\gamma_{2,2} X_{t-1}{ }^{\left(M^{2}\right)}+\gamma_{2,3} X_{t-1}{ }^{\left(M^{3}\right)}+\omega_{t}^{\left(M^{1}\right)}
\end{gathered}
$$

i.e. months $10,11 \& 12$

- In the second month, the representation is the same as in the first one, but the first month of the current quarter $\left(X t^{\left(M^{1)}\right)}\right.$ is added to the model specification:

$$
\begin{aligned}
& Y_{t}^{(Q)}=\beta_{1}{ }^{\left(M^{2}\right)}+\varphi_{1} Y_{t-1}{ }^{(Q)}+\gamma_{1,1} X_{t}{ }^{\left(M^{1}\right)}+\gamma_{2,2} X_{t-1}{ }^{\left(M^{2}\right)}+\gamma_{2,3} X_{t-1}{ }^{(M} \\
&+\omega_{t}{ }^{\left(M^{2}\right)}
\end{aligned}
$$

i.e. months, $1,11 \& 12$

- In the third, two months of the current quarter $(t)$ and one month of the previous quarter $(t-1)$ are included ( 3 months of the variable $X$ in total):

$$
\begin{aligned}
& Y_{t}^{(Q)}=\beta_{1}^{\left(M^{3)}\right.}+\varphi_{1} Y_{t-1}^{(Q)}+\gamma_{1,1} X_{t}^{\left(M^{1}\right)}+\gamma_{1,2} X_{t}^{\left(M^{2}\right)}+ \\
& \gamma_{2,3} X_{t-1}^{\left(M^{3)}\right.}+\omega_{t}^{\left(M^{3}\right)}
\end{aligned}
$$

\section{i.e. months $1,2 \& 12$}

where $\omega t^{(M j)}$ for $j=1,2$ or 3 , is the error term of the regressions.

Also, in U-MIDAS, the weights assigned to each month are completely data-driven, reflecting the concept that each month of data has different importance in forecasting GDP. U-MIDAS has another appealing feature fo forecasting in short-term horizon. Namely, unlike bridge equations approach, for instance, it does not necessitate a forecast of missing months and consequently does not require any assumptions about the development of the indicators in the following months (adaptation from Leboeuf and Morel 2014).

\section{Evaluation of the models by means of empirical comparison}

Empirical comparison is supposed to be a standard when evaluating different models regarding their usefulness in regular projection rounds. Consequently, in the pages that follow we are assessing how the results of the two modelling frameworks will generalize to the independent data set. Put differently, we attempt to discover whether the relatively simpler U-MIDAS could allow for some predictive gains over the MF-VAR model at very near term.

Moreover, in practice, when deciding to establish some model as a tool in the regular economic analysis, besides low RMSE, model's forecasts smooth response to news is also a very relevant issue. To this end, we are looking for a strong evidence in improving forecasting performance in both, a "normal" period like 2016, and a crisis year like $2017^{5}$. Therefore, the model is evaluated based on both, its ability to approximate history, and its usefulness to capture the turning points.

\subsection{Main findings from the empirical exercise}

This section outlines the average performance of the employed modelling frameworks. To this end, we report the RMSE performance of the MF-VAR for nowcasting quarterly GDP growth at one period ahead horizon, against the U-MIDAS pooled forecasts. In addition, we report the combination forecast of these two models using inverse mean square error (IMSE) weighting scheme ${ }^{6}$. The RMSE result for the benchmark bridge equations model is presented as well.

The results aligned with the bridge equations model, U-MIDAS pooled forecasts and the MF-VAR individual forecasts, as well as their combination forecasts are summarized in the Table 1 below. The results, are obtained recursively, based on log difference approximation as well as on seasonally adjusted figures, for the evaluation sample 2015 Q4 2017 Q4 (third months of the quarter).

Considering the fact that we evaluate the models not only statistically but also regarding their ability for pseudo out-of-sample projecting of turning points, we might say that the benchmark model, (i.e. bridge equations framework) is outperformed by the mixedfrequency approaches in the respective evaluation period because it showed very clear difficulty not only in predicting the cyclical declines, but also in recognizing the presence of negative growth rates. Analogously, in an in-sample nowcasting exercise, the U-MIDAS pooling performs pretty well: it shows a comparable performance with respect to the MF-VAR, although not superior one. Namely, in evaluating forecast performances, we conduct the DieboldMariano test $^{7}$ (Diebold and Mariano, 1995) and compare predictive accuracy between the model nowcasts (for more insight please refer to the Table A.2 in the Appendix). The results obtained indicate that the U-MIDAS regressions show statistically comparable forecasting performance to that of the MF-VAR model. However, MF-VAR delivered better forecasts over the first half of 2017 marked as a period of increased domestic political uncertainty. On the other hand, U-MIDAS produced better predictions than the alternative during the stable times (please refer to Figure 1). 
Table 1. Overview on nowcast pooling based on twelve single indicator bridge equations,U-MIDAS regressions ${ }^{8}$, MF-VAR individual nowcasts and combined forecast

\begin{tabular}{|c|c|c|c|c|c|}
\hline & $\begin{array}{c}\text { actual GDP } \\
\text { growth }\end{array}$ & $\begin{array}{l}\text { Bridge equations } \\
\qquad \mathrm{hm}=1\end{array}$ & $\begin{array}{c}\text { U-MIDAS } \\
\mathrm{hm}=1\end{array}$ & $\begin{array}{c}\text { MF-VAR } \\
\mathrm{hm}=1\end{array}$ & $\begin{array}{c}\text { Combined } \\
\text { (U-MIDAS \& MF-VAR) } \\
\mathrm{hm}=1\end{array}$ \\
\hline 2015Q4 & 0.82 & 0.14 & 0.62 & 1.46 & 1.20 \\
\hline 2016Q1 & 0.37 & 0.47 & 0.75 & -0.25 & 0.06 \\
\hline 2016Q2 & 0.18 & 0.77 & 0.77 & -0.83 & -0.33 \\
\hline 2016Q3 & 0.89 & 0.68 & 0.92 & 1.14 & 1.07 \\
\hline 2016Q4 & 1.23 & 0.42 & 0.67 & 1.15 & 1.00 \\
\hline 2017Q1 & -2.09 & 0.05 & -0.06 & -0.36 & -0.27 \\
\hline 2017Q2 & -0.88 & 1.70 & 1.88 & -1.03 & -0.12 \\
\hline 2017Q3 & 1.72 & 1.17 & 1.41 & 2.45 & 2.12 \\
\hline 2017Q4 & 2.10 & -0.10 & -0.10 & 0.51 & 0.32 \\
\hline RMSE & & 1.41 & 1.39 & 0.94 & 0.93 \\
\hline
\end{tabular}

Source: Authors' calculations

Figure 1. Actual versus predicted quarterly GDP growth (obtained recursively, based on log difference approximation, seasonally adjusted figures)

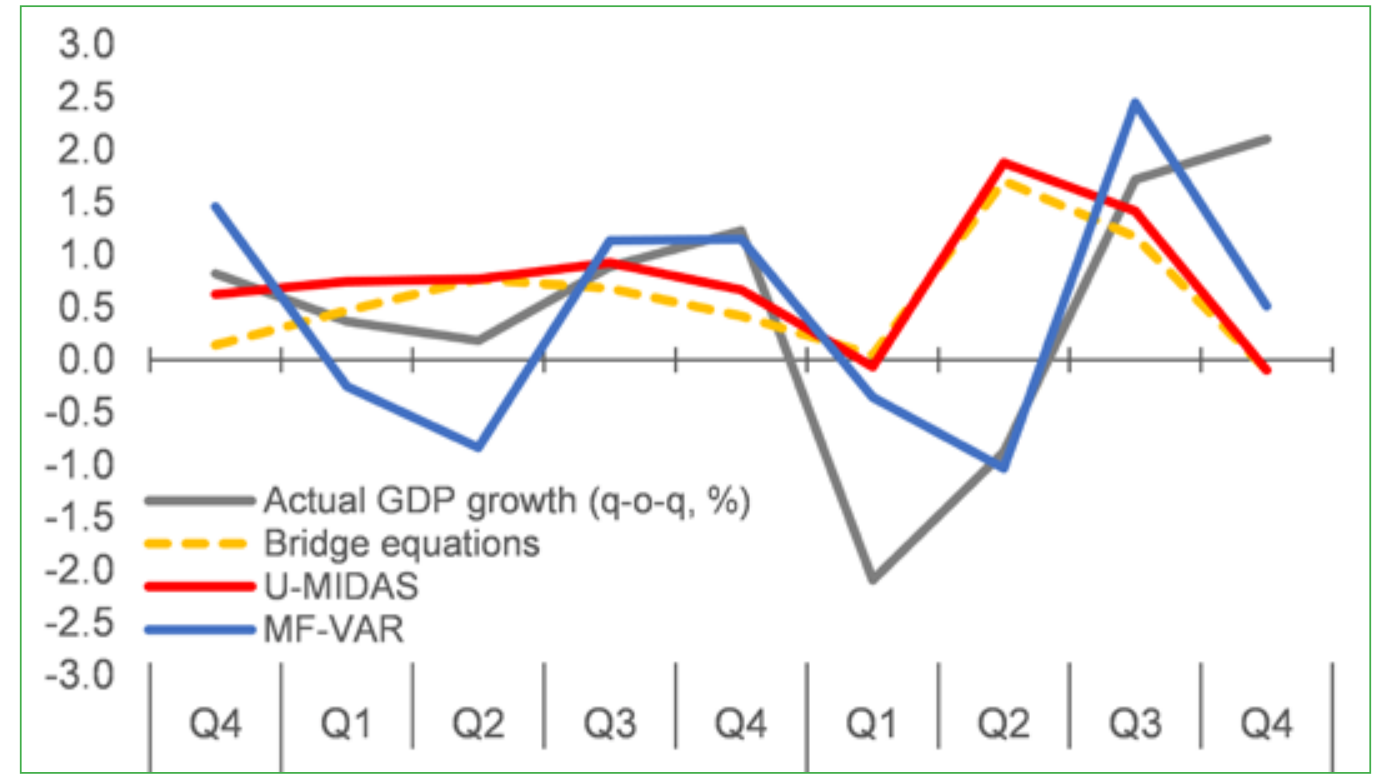

Source: Authors' calculations

Consequently, it is interesting to point out that the two approaches (i.e. U-MIDAS and MF-VAR) are in fact complementing, since in general, their combined forecast exhibit a tendency to be superior in forecasting the turning points of the business cycle (please see Figure 2). 
Figure 2. Actual versus combined forecast of quarterly GDP growth (obtained recursively, based on log difference approximation, seasonally adjusted figures)

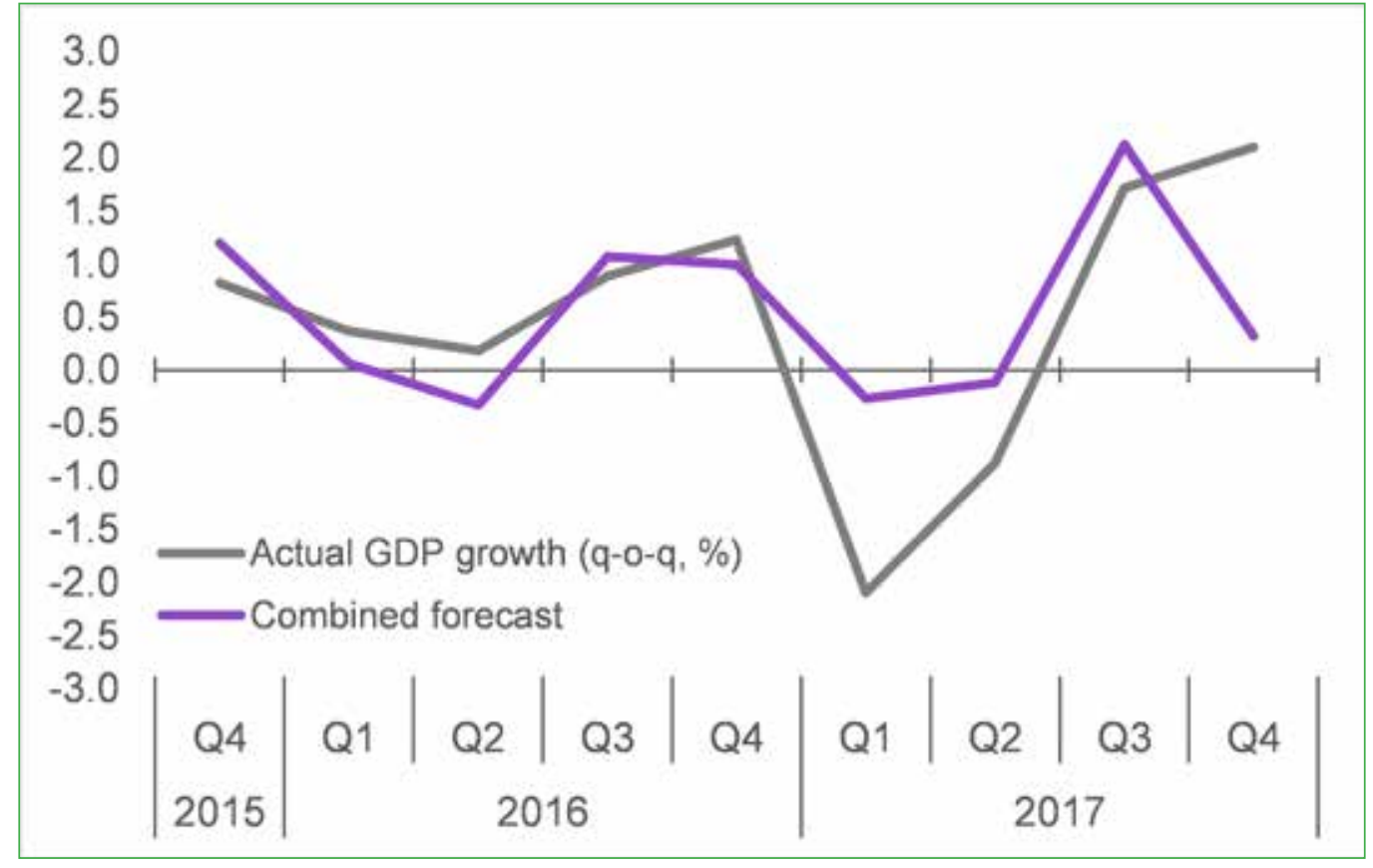

Source: Authors' calculations

\section{Conclusion}

Evaluation of the models regarding their utility in regular forecasting rounds should be backed-up by an empirical comparison. Analysed from the perspective of our central research question, the study results show that there is no statistically significant difference in the forecasts produced by the two mixed-frequency approaches for the pseudo-out-of-sample period.

More precisely, the results obtained point out that the U-MIDAS regressions show statistically comparable forecasting performance to that of MF-VAR model and that the two approaches are actually reciprocal, given that their combined forecast in general shows a superiority in projecting the turning points of the business cycle. However, in our empirical exercise, the
MF-VAR model delivered more accurate predictions, in times of increased uncertainty, when reliable assessments of the current situation are most needed. However, this particular annotation should be taken as indicative rather than definitive, given the relatively short test period covering only one such episode. This issue is broadly aligned with one of the main limitations of the study, i.e. lack of longer time series for some of the variables.

The results of this comparative study may be practical to institutional forecasters and economic agents, as information on where the economy is heading is particularly valuable. The MF-VAR and the U-MIDAS are obvious choices for nowcasting in practice. 


\section{Endnotes}

1 Most of them are part of the regular NBRNM's current economic analysis framework. In this regard, speaking generally, the selection of series is based on the existingcontributions in the field literature, as it is also a notion in Abdic et al. (2020). In parallel, the data segment containing quarterly and monthly series is drawn from four main sources: State Statistical Office, National Bank of the Republic of North Macedonia, Eurostat and the European Commission.

2 Considering their estimation simplicity and their transparency, bridge equations are extensively used in policy institutions, in particular, central banks.

3 Forecasts of the high frequency indicators within the bridge equations framework are obtained by using some particular time series models (best- fitting ARIMA models based on minimum AIC criterion, limiting the number of AR and MA terms to 3 ). The subsequent forecast values are aggregated and added into the bridge equations in order to get the low-frequency variable forecast.

4 That has been pioneered by Ghysels, Santa-Clara, and Valkanov (2004).

5 Domestic political uncertainty-related risk stands out in this year's risk landscape.

6 We employ the inverse value of the MSE in order to penalise the model that is associated with greater uncertainty.

7 We apply quadratic loss function for the DieboldMariano test.

8 We consider simple mean as a combination scheme the most exploited method in the literature.

\section{References}

Aastveit, K.A., Foroni, C. and Ravazzolo, F. 2016. Density forecasts with MIDAS models.Journal of Applied Econometrics, 32(4): 783-801.

Abdić, A., Resić, E., Abdić, A., and Rovčanin, A. 2020. Nowcasting GDP of Bosnia and Herzegovina: a comparison of forecast accuracy models. South East European Journal of Economics and Business, 15 (2): 1-14.

Andreou, E., Ghysels, E. and Kourtellos, A. 2013. Should Macroeconomic Forecasters Use Daily Financial Data and How?.Journal of Business \& Economic Statistics, 31(2):240-251.

Andreou, E., Gagliardini, P., Ghysels, E. and Rubin, M. 2019. Inference in Group Factor Models With an Application to Mixed-FrequencyData.Econometrica, 10.3982/ ECTA14690, 87(4):1267-1305.

Bai, J., Ghysels, E. and Wright, J.H. 2013. State Space Models and MIDAS Regressions.Econometric Reviews, 32(7):779-813.

Bańbura, M., Giannone, D., Modugno, M. and Reichlin, L. 2013. Now-casting and the real-time data flow, Working Paper SeriesNo. 1564, European Central Bank.

Barsoum, F., and Stankiewicz, S. 2013. Forecasting GDP Growth Using Mixed-Frequency Models With Switching Regimes. Working Paper Series of the Department of Economics 2013-10, University of Konstanz.

Chiu, C.W., Eraker, B., Foerster, A.T., Kim, T.B. and Seoane, H.D. 2011. Estimating VAR's Sampled at Mixed or Irregular Spaced Frequencies: A bayesian approach, Research Working Paper RW 11-11, Federal Reserve Bank of Kansas City.

Clements, M. and Galvão, A. 2008. Macroeconomic forecasting with mixed frequency data: forecasting output growth in the United States. Journal of Business and Economic Statistics, 26:546-554.

Clements, M. and Galvão, A. 2009. Forecasting US Output Growth Using Leading Indicators: An Appraisal Using MIDAS Models. Journal of Applied Econometrics,24(7): 1187-1206.

Diebold, F. X. and Mariano,R. S. 1995. Comparing predictive accuracy.Journal of Business \& Economic Statistics, 13:253-263.

Drechsel, K. and Scheufele, R. 2012. Bottom-up or Direct? Forecasting German GDP in a Data-rich Environment, Working Papers, No 2012-16, Swiss National Bank.

Duarte, C. 2014. Autoregressive augmentation of MIDAS regressions. Working Papers w201401, Banco de Portugal, Economics and Research Department.

Ferrara, L., Marsilli, C. and Ortega, J-P. 2014. Forecasting Growth During the Great Recession: Is Financial Volatility the Missing Ingredient?.Economic Modelling, 36: 44-50,Working Paper No. 454, Banque de France. 
Foroni, C. and Marcellino, M. 2013. A survey of econometric methods for mixed-frequency data. Department of Economics 2013/02. European University Institute.

Foroni, C. and Marcellino, M. 2014. A comparison of mixed frequency approaches for nowcasting Euro area macroeconomic aggregates.International Journal of Forecasting, 30(3):554-568.

Foroni, C., Marcellino, M. and Schumacher, C. 2011. U-MIDAS: MIDAS regressions with unrestricted lag polynomials. Discussion Paper Series 1: Economic Studies 35, Deutsche Bundesbank, Research Centre.

Foroni, C., Marcellino, M. and Stevanovic, D. 2018. Mixed Frequency Models with MA Components. Deutsche Bundesbank Discussion Paper No. 02/2018.

Ghysels, E., Santa-Clara, P. and Valkanov, R. 2004. The MIDAS Touch: Mixed Data Sampling Regression Models. CIRANO Working Papers 2004s-20, CIRANO.

Ghysels, E., Santa-Clara, P. andValkanov, R. 2005.There is a riskreturn trade-off after all. Journal of Financial Economics, 76:509-548. doi:10.1016/j.jfineco.2004.03.008

Ghysels, E., Santa-Clara, P. and Valkanov, R. 2006. Predicting volatility: getting the most out of return data sampled at different frequencies. Journal of Econometrics, 131:59-95.

Kapetanios, G., Marcellino, M. and Petrova, K. 2018. Analysis of the most recent modelling techniques for big data with particular attention to Bayesian ones. Eurostat Statistical Working Papers, 2018 edition.
Kuzin, V., Marcellino, M. and Schumacher, C. 2011. MIDAS vs. Mixed frequency VAR: Nowcasting GDP in the euro-area. International Journal of Forecasting, 27(2):529-542.

Leboeuf, M. and Morel, L. 2014. Forecasting Short-Term Real GDP Growth in the Euro Area and Japan Using Unrestricted MIDAS Regressions. Discussion Papers, Bank of Canada.

Marsilli, C. 2014. Mixed-Frequency Modeling and Economic Forecasting. Economics and Finance. Université de Franche-Comté, 2014. English. NNT: 2014BESA2023. tel-01645421.

Mikosch, H. and Neuwirth, S. 2015. Real-time forecasting with a MIDAS VAR. BOFIT Discussion Papers 13/2015, Bank of Finland, Institute for Economies in Transition.

Mittnik, S. and Zadrozny, P.A. 2004. Forecasting Quarterly German GDP at Monthly Intervals Using Monthly IFO Business Conditions Data. CESifo Working Paper Series No. 1203, CESifo Group Munich.

Mariano, R. and Murasawa, Y. 2010. A Coincident Index, Common Factors, and Monthly Real GDP, Oxford Bulletin of Economics and Statistics, 72(1):27-46.

Schorfheide, F. and Song, D. 2015. Real-Time Forecasting With a Mixed-Frequency VAR.Journal of Business \& Economic Statistics, 33(3):366-380. 


\section{APPENDIX}

Table A.1. Employed variables and the corresponding publication lags

\begin{tabular}{lll}
\hline Main releases & Publishing lag & Frequency \\
\hline Number of employees - Total -Industry & 1 month & monthly \\
Turnover recorded in capital goods industries & 2 months & monthly \\
Industrial production index - Total - Germany & 1 month & monthly \\
Manufacture of motor vehicles, trailers and semi-trailers in EU-28 & 1 month & monthly \\
PPI - Exporting industries (PPI=Producer Price Index) & 1 month & monthly \\
Hours worked - Construction & 2 months & monthly \\
Industrial production index - Manufacture of other non-metallic mineral products & 1 month & monthly \\
M2-Denar part & 1 month & monthly \\
Real average monthly net-wage & 2 months & monthly \\
Tourism-overnight stays & 2 months & monthly \\
EC ESI-Macedonia (EC ESI=European Commission Economic Sentiment Indicator) & 1 month & Monthly \\
EC ESI-Germany (EC ESI=European Commission Economic Sentiment Indicator) & 1 month & Monthly \\
Gross Domestic Product at constant prices (millions of Denar) & &
\end{tabular}

Notes: The publication lags reflect the number of missing values at the end of each quarter

Table A.2. Diebold-Mariano test (HLN adjusted)

\section{U-MIDAS vs. MF-VAR}

Null hypothesis: Both forecasts have the same accuracy

\begin{tabular}{ccccc}
\hline Accuracy & Statistic & $<$ prob & $>$ prob & $<$ prob \\
\hline Abs Error & 0.762745 & 0.4675 & 0.7662 & 0.2338 \\
Sq Error & 1.208683 & 0.2613 & 0.8694 & 0.1306 \\
\hline
\end{tabular}

\section{Bridge equations vs. U-MIDAS}

Null hypothesis: Both forecasts have the same accuracy

\begin{tabular}{ccccc}
\hline Accuracy & Statistic & $<$ prob & $>$ prob & $<$ prob \\
\hline Abs Error & 1.14736 & 0.2844 & 0.8578 & 0.1422 \\
Sq Error & 0.321752 & 0.7559 & 0.6221 & 0.3779 \\
\hline
\end{tabular}

\section{Bridge equations vs. MF-VAR}

Null hypothesis: Both forecasts have the same accuracy

\begin{tabular}{ccccc} 
Accuracy & Statistic & $<>$ prob & $>$ prob & $<$ prob \\
Abs Error & 1.124426 & 0.2934 & 0.8533 & 0.1467 \\
Sq Error & 1.439139 & 0.1881 & 0.906 & 0.094 \\
\hline
\end{tabular}

Source: Authors' calculations 